\title{
Optical Internet of Things within 5G: Applications and Challenges
}

\author{
Shivani Rajendra Teli ${ }^{1}$, Stanislav Zvanovec ${ }^{1}$, and Zabih Ghassemlooy ${ }^{2}$ \\ ${ }^{1}$ Dept. of Electromagnetic Field, Faculty of Electrical Engineering, Czech Technical University in Prague, Prague, 16627, Czech Republic, \\ ${ }^{2}$ Optical Communications Research Group, Faculty of Engineering and Environment, Northumbria University, Newcastle-upon-Tyne, NE1 8ST, \\ $U K$ \\ \{telishiv;xzvanove\}@fel.cvut.cz,z.ghassemlooy@northumbria.ac.uk
}

\begin{abstract}
The fifth generation (5G) telecommunications standards are being developed to meet the growing demands for high-speed wireless networks (i.e., few tens of Gigabits per second). The 5G standard stems largely from an increasing number of users and plethora of different devices, collectively referred to as smart devices, connecting to a network as part of Internet-of-Things (IoT). A few potential technologies have emerged for $5 \mathrm{G}$ such as millimeter waves, massive multiple-input multiple-output, and small cell communications. Although these technologies would satisfy the requirements of $5 \mathrm{G}$, there is a complementary alternative wireless technology of optical wireless communications (OWC), which is being considered. As part of OWC, visible light communications (VLC) and optical camera communications (OCC) are the most attractive options for $5 \mathrm{G}$ networks and beyond. VLC with huge frequency spectrum integrated with IoT can open up a wide range of indoor and outdoor applications as part of future smart environments. This paper provides an overview of the all-optical IoT (OIoT) focusing on VLC and OCC based potential applications and challenges as part of 5G standards.
\end{abstract}

Keywords-Optical wireless communications (OWC); visible light communications (VLC); optical camera communications (OCC); Internet of things (IoT); light emitting diodes (LEDS).

\section{INTRODUCTION}

The fifth generation (5G) wireless network is being developed as a new platform for mobile networks, rather than just extended the capacity and reliability of the existing 4G [1] [2]. As such, the focus in the $5 \mathrm{G}$ standard is on capacity enhancement, massive connectivity, and ultra-high reliability (low latency) [1]. They stem largely from an increasing number of users and smart devices connecting to a cellular network in the Internet-of-Things (IoT) domain as part of smart cities. Given the rise of IoT, $5 \mathrm{G}$ is well positioned to be the standard framework for global communication platforms, which support real-time interactions with wireless sensor networks and devices at a large scale. While $5 \mathrm{G}$ is still yet to be finalized, some of the candidate technologies have emerged: A) Millimeter wave (MMW) technology, which is intended to dramatically increase the spectrum efficiency, especially base stations, by utilizing a carrier frequency in the range from 30 $300 \mathrm{GHz}$ [3]. Recently, the mmMAGIC project [4] has been involved in research and development of novel radio access technologies at the MMW frequency bands of 6-100 GHz with the aim of increasing the capacity and data rates. Thus, outlining a comprehensive overview of the key research areas, channel modelling, network architecture, use case and challenges of MMW for 5G. B) Massive multiple-input multiple-output (MIMO), which could provide hundreds of ports for both uplink and downlink utilizing dozens of antennas to extend the capacity by a factor of 22 or more. C) Small cells based base stations, which offer the relay-based capability for signal transmission between MMW base stations and the mobile users. The requirements in communication systems design to support IoT are as follows (i) less complexity and cost-effectiveness; (ii) resource availability; (iii) quality of service and reliability; (iv) transmission range; (v) safety and (vi) low power consumption.

IoT represents the network of physical devices, sensors within the smart environments and their interconnectivity enables objects to communicate and exchange data between themselves [5]. As the IoT paradigm opens the doors to innovations, which contribute to interactions between objects and humans, it enables the realization of smart cities, infrastructures, and services for enhancing the quality of life and improving better utilization of resources. The radio frequency (RF) and Android-based IoT has been implemented for home automation systems as part of smart home environments [6]. A Raspberry Pi camera based body detection using passive infrared sensors and computer vision for security application was proposed in [7]. A survey on IoT application for outdoor environments such as traffic surveillance and accident detection system, which provides a smart way to handle traffic and solve concerned problems, was presented in [8].

Over the last decade, an increasing number of multimediacapable and Internet-connected mobile devices have demanded highly reliable and high-speed connectivity for both indoor and outdoor applications [1]. With the introduction of IoT, these demands are becoming even higher. So far, conventional RFbased communications have been the dominant technology for meeting these demands by employing complex coding and multilevel modulation schemes as well as spatial diversity. However, the RF spectrum has largely reached its saturation level with slow-down in the achievable spectrum efficiency [5]. 
As a potential alternative to congested RF-based communication systems, optical wireless communication (OWC) is envisioned as a next-generation communications system, which could deliver very high-speed and reliable data transmission in a specific application in both indoor and outdoor environments $[9,10]$. This paper aims to provide an overview of advancements, applications, issues and challenges in OWC-based IoT as part of 5G wireless networks, which is termed as optical IoT (OIoT).

The remainder of the paper is organized as follows. Section II introduces OWC, while Section III describes the proposed OIoT. Section IV describes the OIoT - applications and challenges and finally the conclusions are drawn in Section $\mathrm{V}$.

\section{OPTICAL WIRELESS COMMUNICATIONS}

The extensive deployment of smart devices within smart environments (i.e., homes, offices, industrial halls and deviceto-device (D2D) communications) as of the emerging IoT represents a major challenge for communication networks service providers in order to provide cost-effective and high quality of service wireless connectivity [11]. In parallel to the developments in the RF domain, there is the potential to adopt a complementary wireless technology of OWC in certain areas where connectivity can readily be established [9]. In OWC, the three main bands of ultraviolet, infrared and visible light can be utilized. Within the latter two bands, visible light communications (VLC), free space optics (FSO) and optical camera communications (OCC), see Fig. 1, can be potentially considered as part of the $5 \mathrm{G}$ networks for the realization of IoT [11].

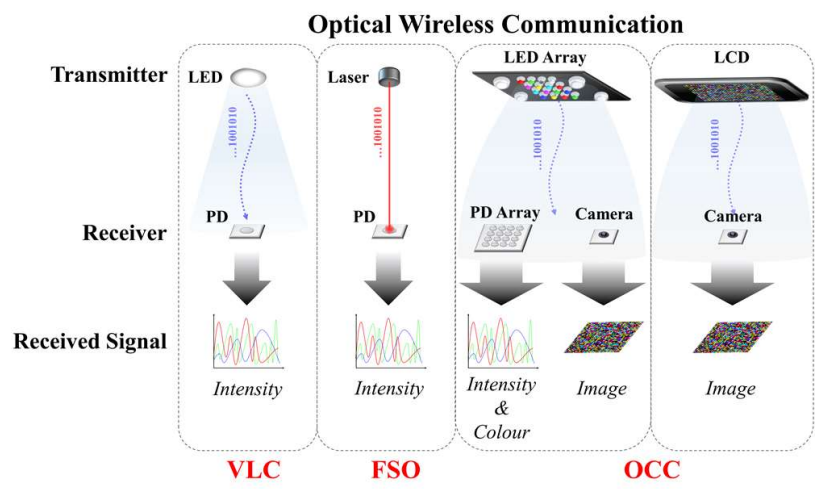

Fig. 1. Overview of OWC schemes.

VLC is an emerging technology, which has been proposed for the $5 \mathrm{G}$ networks. It utilizes light-emitting diode-based lighting fixtures and photodetectors (PDs) to simultaneously provide data communications and illumination in indoor environments with possible extension to outdoor areas as well [12]. Note that, the visible wavelength band of $\sim 370-780 \mathrm{~nm}$ provides a massive bandwidth of $\sim 400 \mathrm{THz}$, which is 10,000 times larger than the RF bandwidth [13]. In addition, the VLC technology offers inherent security at the physical layer (PHY), immunity to the RF electromagnetic interference, and free licensing [10]. The light emitting diodes (LEDs) offer several benefits over existing lighting infrastructures, such as lower power consumption, longer life expectancy, higher energy efficiency, reduced maintenance, lower heat generation characteristics and fast switching speed (orders of magnitude higher) [10]. Therefore, it can be said, "the VLC technology utilizing smart LEDs could join the dots of IoT". The VLC can enable true IoT as most consumer smart devices come with LEDs and cameras, which can be used for data communications and indoor localization [14].

On the other hand, over the past few years, we have seen smart devices with built-in high-resolution complementary metal-oxide-semiconductor (CMOS) cameras [15]. These CMOS cameras are capable of capturing high-resolution videos with a resolution of at least $1280 \times 720$ pixels and a capture rate of $30 \mathrm{fps}[16,17]$, which are more than adequate for low-speed applications. Due to the large scale and increasing availability of mobile phones, smartphone VLC can be attractive, as nearly all mobile users effectively carry and regularly use camerabased optical receivers. Not only smartphones but also the majority of new generation smart devices have built-in CMOS cameras, providing the ability to capture photos and videos as well as being used for data communications (low-speed), indoor localization and range findings $[16,18]$. The smartphone or camera-based VLC has been studied within the framework of OWC and considered as a candidate for IEEE802.15.7rl standard and is referred to as OCC $[19,20]$. OCC represents an extension of VLC with the advantage of no additional hardware to establish D2D communications at low data rates and indoor positioning [20]. Unlike conventional VLCs, which employ PDs as the receiver, in OCC a mobile phone CMOS camera is used as the receiver [17]. That is, OCC captures twodimensional data in the form of image sequences, thus is able to transmit more information compared to photodetector-based VLCs. The OCC technology is making remarkable progress in the key application as part of the fourth industrial revolution i.e., IoT, smart vehicles, etc. [21, 22].

\section{OPTICAL INTERNET OF THINGS}

VLC with a huge frequency spectrum integrated with IoT can open up a wide range of applications for both indoor and outdoor environments [23]. Fig. 2 illustrates the key features of OIoT, which are outlined below:

1) Capacity and efficiency: Due to the increasing demand for high-speed wireless services, the RF spectrum $(3 \mathrm{kHz}-$ $300 \mathrm{GHz}$ ) is being congested, thus resulting in the bandwidth bottleneck. The visible light spectrum $(400 \mathrm{THz}$ to $780 \mathrm{THz}$ ) offers bandwidth orders of magnitude higher the RF, which can be effectively utilized in IoT networks [24]. In addition, small and compact VLC modules for OIoT can be easily implemented into the existing lighting infrastructure. LEDs represent green-lighting devices, which are being widely used at a global level because of the high power efficiency of $80 \%$ compared to the traditional lights [25]. A recent report from the U.S. 


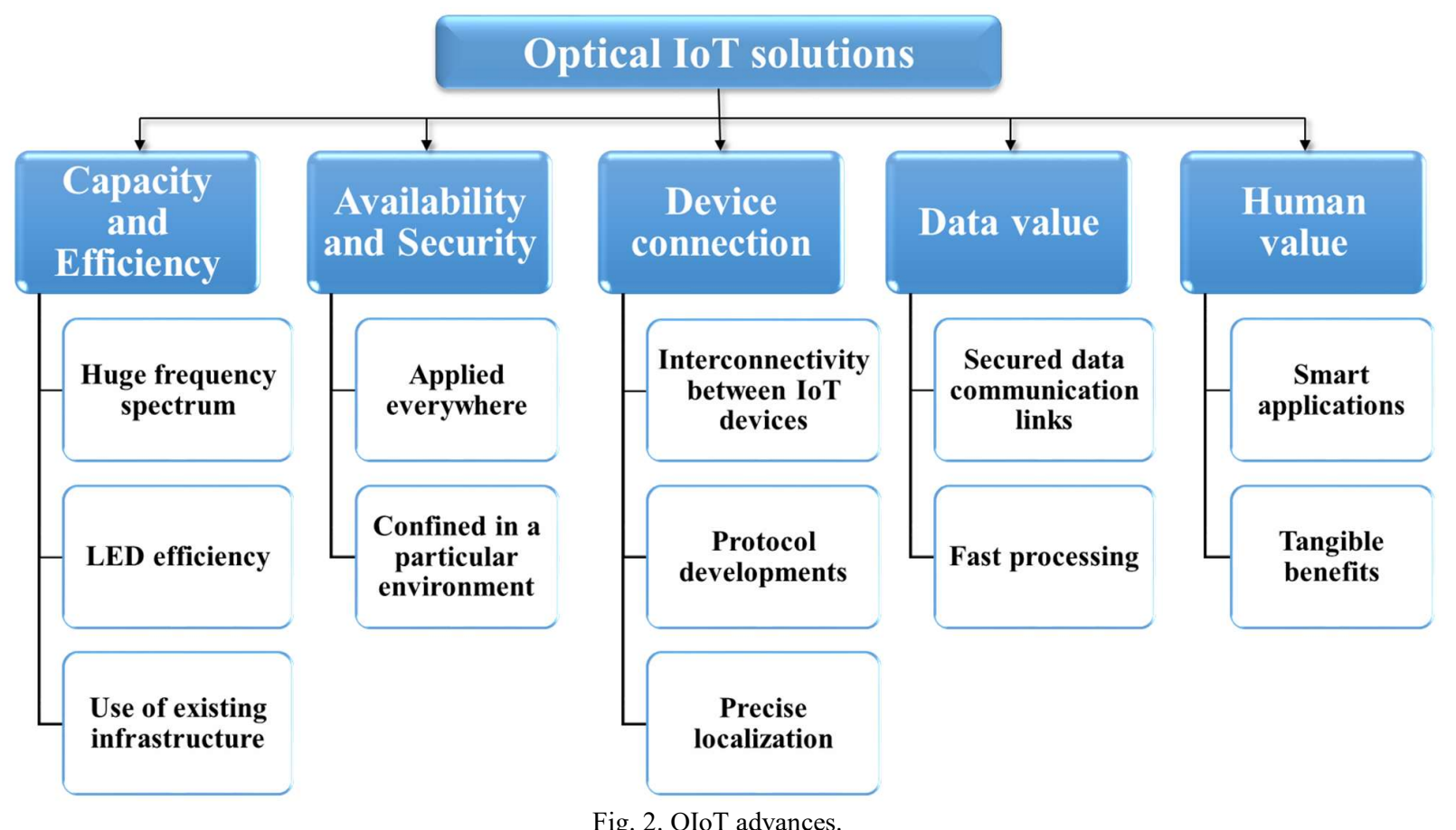

Fig. 2. OIoT advances.

Department of Energy states, by the year of 2025, it is possible to save energy by up to 217 terawatt-hours (TWh) by using LED-based lighting technology [26].

2) Availability and security: The OIoT system based on visible lights can be designed by re-using the ubiquitous lighting infrastructure with only a few additional modules (modulation unit, digital-to-analog converter, and driving circuit), which can be included in the LED lighting systems. Due to the rapid growth of the LED industry, it is expected that the cost of VLC transceivers will be reduced.

Security is an important issue in RF communications since RF signal can penetrate walls and other objects, thus compromising on the link security at the physical level. In contrast to RF, light signals can be confined within a specific well-defined area both indoor and outdoor, thus making eavesdropping almost impossible unless the receiver is within the field of view of the transmitter. In addition, light generated by LEDs are safe for the environment provided the illumination level is below the recommended standard.

3) Device connection: The interconnectivity between various OIoT devices can be maintained by means of adopting the most suitable handover algorithm to ensure seamless communications while mobile devices are moving around within a certain transmission range of course [27]. For longer range, relayed based OIoT could be adopted. Moreover, protocol developments for the PHY, Media Access Control (MAC) and upper layer design to optimize link reliability are studied under IEEE 802.15.7 [28].

On the other hand, a combination of VLC and OCC systems can also be leveraged for indoor localization with very high positioning accuracy [29]. Indoor positioning and localization are achieved by using the LED lights similar to the global positioning system. The experiment results in [30] demonstrate a mean positioning error of less than $1.7 \mathrm{~cm}$ using indoor VLC based positioning system based on orthogonal frequency division multiplexing access.

4) Data value: Secured data communication links can be formed using visible light as the carrier signal in OIoT. The new, fast and efficient adaptation techniques for VLC have been studied in [31] to improve the receiver signal-to-noise (SNR) ratio and to reduce the required time to estimate the position of the VLC receiver.

5) Human value: Developing cost-effective, environmentally friendly and efficient OIoT within smart environments (i.e., homes, hospitals, industries, cities, etc.) would be possible, which offers higher speed and safer communications.

\section{OIOT: APPLICATIONS AND CHALLENGES}

The OIoT solutions mentioned in Section III can help to yield various indoor and outdoor applications. Using advanced techniques such as multicarrier modulation, wavelength division multiplexing, and equalization the data rate can be increased to a few Gbps [24]. Machine-to-machine (M2M) communications based on MIMO OWC was demonstrated in [32] as field trials at the BMW's robot testing facility. Whereas, efficient D2D communications over a transmission span of $1 \mathrm{~m}$ and over an extended distance up to $3.5 \mathrm{~m}$ using an optical repeater in an indoor environment was investigated in [33].

Fig. 3 shows applications of OIoT within different environments. The high-speed VLC can initially be used in places, where Wi-Fi connectivity is limited or is poor i.e., 


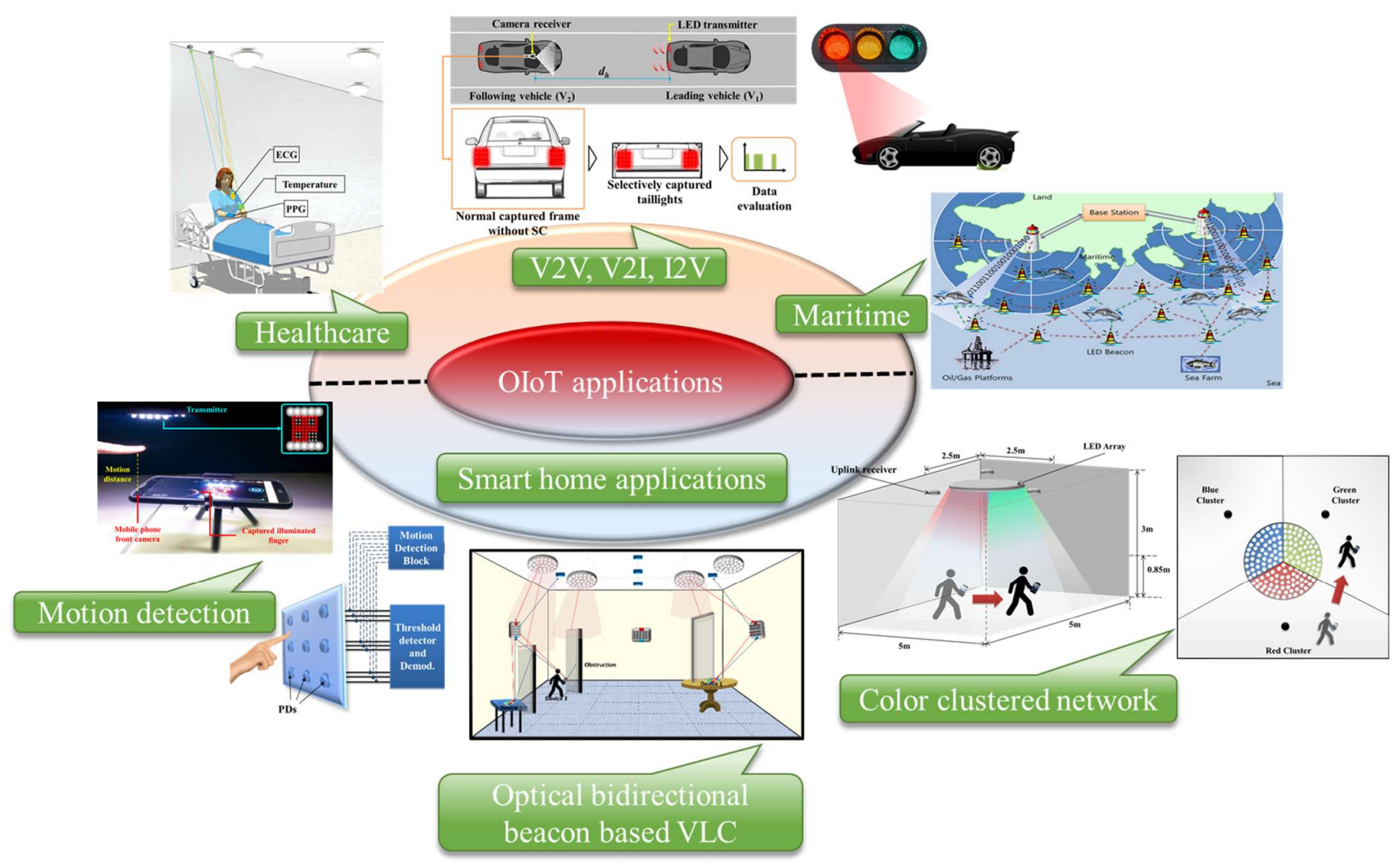

Fig. 3. OIoT applications.

hospitals (connectivity of modern medical instruments, on body placed sensors termed as wireless body area network, WBAN, [34] etc.) or airplanes. Dense urban environments, intelligent transportation systems (i.e., car to car communications), submarines and remotely operated underwater vehicles can benefit from the connectivity as well as links within chemical and power plants where RF use is prohibited [35]. To this end, extensive works have been reported by extending the point-topoint communication links mentioned in the IEEE 802.15.7 standard [36]. In this respect, resource allocation was proposed in [37] and a user allocation scheme in [38] to address multiuser bidirectional transmission scenarios in a VLC environment. An optical signal transmission-based body area network, termed optical body area network (OBAN), as an alternative solution to RF-based BAN for transmitting multiple patients' vital signs was proposed in [34]. Underwater and maritime VLC systems using blue lights are also being investigated as an alternative wireless technology for exploration of oceans [39].

Recently, an IoT device management protocol over VLC networks using IPv6 over a low power wireless personal area network was proposed in [40]. However, the proposed protocol was implemented only on devices supporting Java Virtual Machine. In [41], a visible light based time synchronization protocol for IoT termed as PSync was introduced. The research stated that the energy consumed for a single round of synchronization based on PSync can be as low as $19 \%$ of the energy needed to receive a small packet ( 1 byte) using IEEE 802.15.4 radio. The solution to indoor localization for various location-based IoT applications with acceptable simplicity, robustness, accuracy and responsiveness still needs to be explored. In [42], Foglight: visible light-enabled indoor localization for low-power IoT devices using a hybrid VLCWiFi network was investigated. The PHY and MAC used to optimize the link reliability of a short-range VLC are given in IEEE 802.15.7 [28]. A VLC system employing spectral amplitude coding optical code division multiple access was proposed to improve the flexibility in channel allocation, ability to access asynchronously, enhanced privacy and increased network capacity [43].

OCC using image sensor in a camera can detect signals from LED displays and screens acting as transmitters in public areas such as airports and train stations, shopping malls and outlets, where merchandise and advertisement information can be broadcasted to customers using signage [44]. Recently, within smart home environments, OCC and VLC based motion detection to transmit the control signals $[16,45]$, optical beacon based VLC [46] and color clustered networks [38] were studied. Image processing is of paramount importance in OCC for demodulation of the received data in the form of captured image frames. Therefore, it is necessary to have a robust and reliable image processing algorithms and schemes. In recent years, neural networks $(\mathrm{NN})$ have attracted much attention to solving 
complex problems related to image recognition using an intelligent machine-learning technique. NNs are adopted in order to identify objects' shape in images, transcribe speech into text, match classified items, and select relevant results of a search [47]. In addition to image processing, using an artificial neural network (ANN) equalizer in VLC has resulted in increased data rates by reducing the influence of multipath induced intersymbol interference [48]. Authors in [49] investigated an NN based event processing for an Internet of Multimedia Things (IoMT). NN was used for processing of image events to extract features and for object detection to reduce time complexity. $\mathrm{NN}$ in the form of trained neurons also plays an important role in motion detection over the existing indoor OCC links as was demonstrated in [50]. Design of deep learning based transceiver for multi-colored VLC systems was proposed in [51]. In the context of VLC, an optical neuron concept was implemented based on the multilayer perceptron scheme [52] to equalize the incoming signals based on the received samples in the context of the classification method.

Viable applications of OCC within IoT based networks can be IoT and smart home networks based on LED lights, mobile atto-cell, vehicle-to-everything (V2X), smart surveillance systems, etc. Furthermore, the OCC is provided with an add-on and interesting functionality of motion detection [16] along with data communications and illumination, termed as motion over the camera, which can also serve as a control of signal communications within IoT networks. Considering various camera capturing techniques, a selective capture (SC) based optical camera vehicle-to-vehicle (V2V) communications was proposed in [21]. The SC technique was designed and implemented to address one of the most critical issues of low data rates in OCCs.

Despite various advantages and appealing features as well as several factors that make the VLC deployment, challenging such as connectivity while moving, multiuser support, dimming, shadowing and confinement to a small geographical area. In OCC, the challenges are represented by limited frame rate, synchronization, shot noise, perspective distortions, misalignment and blurry images, and ambient light [24]. These challenges have to be further analyzed in details to strengthen the VLC-OCC implementation within O-IoT networks.

\section{CONCLUSION}

In this paper, we provided an overview of the OIoT networks. The study was mainly focused on VLC and OCC based potential applications and challenges for OIoT networks within $5 \mathrm{G}$ standards. Considering the high potential of optical communication systems in enabling the next generation of wireless communication networks, i.e., 5G, we focused our discussion on the possible applications and challenges within VLC and OCC based OIoT networks to 5G. The proposed OIoT is expected to promote resilient and robust VLC and OCC systems in order to provide high-speed connectivity within the context of greener, cheaper and safer communications technology in smart environments. The recent advancements within VLC and OCC based IoT can pave the way for deployment of OIoT networks in order to realize cost-effective and efficient smart environments. The challenges outlined for VLC and OCC systems as part of the further OIoT networks will be explored in our future research works.

\section{ACKNOWLEDGMENT}

The work is supported by the European Union's Horizon 2020 research and innovation programme under the Marie Sklodowska-Curie grant agreement no 764461 (VisIoN).

\section{REFERENCES}

[1] F. Boccardi, R. W. Heath, A. Lozano, T. L. Marzetta and P. Popovski, "Five disruptive technology directions for 5G, " IEEE Communications Magazine, vol. 52, no. 2, pp. 74-80, 2014.

[2] J. Bloomberg. (2017, Jun 14). "Transformative 5G standards near completion" [Online]. Available: https://www.forbes.com/sites/jasonbloomberg/2017/06/14/transformativ e-5g-standards-near-completion/\#4ea8524a25c4.

[3] A. Nordrum. (2017, Jan 27). Everything You Need to Know About 5G [Online]. Available: http://spectrum.ieee.org/video/telecom/wireless/everything-you-need-toknow-about-5g.

[4] M. Tercero et al., "5G systems: The mmMAGIC project perspective on use cases and challenges between 6-100 GHz," 2016 IEEE Wireless Communications and Networking Conference Workshops (WCNCW), Doha, 2016, pp. 200-205.

[5] H. Aksu, L. Babun, M. Conti, G. Tolomei and S. A. Uluagac, "Advertising in the IoT Era: Vision and Challenges," IEEE Communications Magazine, vol. in press, 2018

[6] P. S. Nagendra Reddy, K. T. Kumar Reddy, P. A. Kumar Reddy, G. N. Kodanda Ramaiah and S. N. Kishor, "An IoT based home automation using android application," 2016 International Conference on Signal Processing, Communication, Power and Embedded System (SCOPES), Paralakhemundi, 2016, pp. 285-290.

[7] N. A. Othman and I. Aydin, "A new IoT combined body detection of people by using computer vision for security application," 2017 9th International Conference on Computational Intelligence and Communication Networks (CICN), Girne, 2017, pp. 108-112.

[8] R. Patel, V. K. Dabhi and H. B. Prajapati, "A survey on IoT based road traffic surveillance and accident detection system (A smart way to handle traffic and concerned problems)," 2017 Innovations in Power and Advanced Computing Technologies (i-PACT), Vellore, 2017, pp. 1-7.

[9] M. Uysal, C. Capsoni, Z. Ghassemlooy, A. Boucouvalas and R. E. Udvary, Optical wireless communications - An emerging technology, Springer, 2016.

[10] Z. Ghassemlooy, N. L. Alves, S. Zvanovec and A. M. Khalighi, Visible Light Communications: Theory and Applications, CRC Press, 2017.

[11] Z. Ghassemlooy, S. Zvanovec, A. M. Khalighi, O. W. Popoola, J. Perez, "Optical wireless communication systems," Optik, vol. 151, pp. 1-6, 2017.

[12] S. Zvanovec, P. Chvojka, A. P. Haigh and Z. Ghassemlooy, "Visible light communications towards 5G," Radioengineering, vol. 24, no. 1, pp. 1-9, 2015.

[13] A. P. Haigh, "Using equalizers to increase data rates in organic photonic devices for visible light communications systems," United Kingdom: Doctoral thesis, University of Northumbria, 2014.

[14] S. Schmid, T. Bourchas, S. Mangold and T. R. Gross, " Linux Light Bulbs: Enabling Internet Protocol Connectivity for Light Bulb Networks," in Workshops on Visible Light Communication Systems (VLCS), 2015.

[15] R. Boubezari, L. H. Minh, Z. Ghassemlooy and A. Bouridane, "Smartphone camera based visible light communication," Journal of Lightwave Technology, vol. 34, no. 17, pp. 4121-4127, 2016.

[16] S. Teli, A. W. Anugrah and H. Y. Chung, "Optical camera communication: Motion over camera," IEEE Communications Magazine, vol. 55 , no. 8 , pp. $156-162,2017$. 
[17] A. W. Cahyadi, H. Y. Kim and H. Y. Chung, "Mobile phone camerabased indoor visible light communications with rotation compensation," IEEE Photonics Journal, vol. 8, no. 2, pp. 1-8, 2016.

[18] T. Nguyen, A. Islam, T. Hossan and M. Y. Jang, "Current status and performance analysis of optical camera communication Technologies for 5G Networks," IEEE Access, vol. 5, pp. 4574-4594, 2017.

[19] M. J. Jang, "IEEE 802.15 WPAN 15.7 Amendment - Optical camera communications Study Group (SG 7a)," 2016. [Online]. [Accessed 15 May 2018].

[20] N. Le and M. J. Jang, "Performance evaluation of MIMO optical camera communications based rolling shutter image sensor," in Eighth Int'l. Conf. Ubiquitous and Future Networks, Vienna, Austria, 2016.

[21] I. Takai, S. Ito, K. Yasutomi, K. Kagawa, M. Andoh and S. Kawahito, "LED and CMOS image sensor based optical wireless communication system for automotive applications," IEEE Photonics Journal, vol. 5, no. $5,2013$.

[22] S. Teli, A. W. Anugrah and H. Y. Chung, "High-speed optical camera V2V communications using selective capture," Photonic Network Communications, pp. - 1-7, 2018.

[23] K. Kadam and R. M. Dhage, "Visible light communication for IoT," in 2nd International Conference on Applied and Theoretical Computing and Communication Technology (iCATccT), Banglore, 2016.

[24] Z. Wang, Q. Wang, W. Huang, Z. Xu, Visible light communications: Modulation and signal processing, John Wiley \& Sons, Nov 29, 2017.

[25] C. W. Chow, C. H. Yeh, Y. F. Liu and Y. Liu, "Improved modulation speed of LED visible light communication system integrated to main electricity network," Electronics Letters, vol. 47, no. 15, pp. 867-868, July 2011.

[26] M. Kavehrad, "Sustainable energy-efficient wireless applications using light," IEEE Communications Magazine, vol. 48, no. 12, pp. 66-73, 2010.

[27] A. M. Vegni and T. D. C. Little, "Handover in VLC systems with cooperating mobile devices," International Conference on Computing, Networking and Communications (ICNC), Maui, HI, pp. 126-130, 2012.

[28] IEEE, "PHY and MAC standard for short-range wireless optical communication using visible light, (Draft 4)," IEEE P802.15.7/D4, pp. 1296, 2010.

[29] Y. Li, Z. Ghassemlooy, X. Tang, B. Lin and Y. Zhang, "A VLC smartphone camera based indoor positioning system," IEEE Photonics Technology Letters, vol. 30, no. 13, pp. 1171-1174, July1, 12018.

[30] B. Lin, X. Tang, Z. Ghassemlooy, C. Lin and Y. Li, "Experimental demonstration of an indoor VLC positioning system based on OFDMA," in IEEE Photonics Journal, vol. 9, no. 2, pp. 1-9, April 2017.

[31] A. T. Hussein, M. T. Alresheedi and J. M. H. Elmirghani, "Fast and efficient adaptation techniques for visible light communication systems," in IEEE/OSA Journal of Optical Communications and Networking, vol. 8, no. 6, pp. 382-397, June 2016.

[32] P. Wilke Berenguer et al., "Optical wireless MIMO experiments in an industrial environment," in IEEE Journal on Selected Areas in Communications, vol. 36, no. 1, pp. 185-193, Jan. 2018.

[33] S. V. Tiwari, A. Sewaiwar, and Y. Chung, " Optical repeater assisted visible light device-to-device communications," International Journal of Electronics and Communication Engineering, vol. 10, no. 2 pp. 206-209, 2016.

[34] D. R. Dhatchayeny, W. A. Cahyadi, S. R. Teli and Y. H. Chung, "A novel optical body area network for transmission of multiple patient vita signs," 2017 Ninth International Conference on Ubiquitous and Future Networks (ICUFN), Milan, pp. 542-544, 2017.

[35] ioTUK, "Li-fi internet of things," [Online]. Available: iotuk, https://iotuk.org.uk/li-fi-internet-things/.

[36] V. Jungnickel et al., "A European view on the next generation optical wireless communication standard," IEEE Conference on Standards for Communications and Networking (CSCN), Tokyo, 2015, pp. 106-111.

[37] A. Sewaiwar, V. S. Tiwari and H. Y. Chung, "Smart LED allocation scheme for efficient multiuser visible light communication networks," Optics Express, vol. 23, no. 10, p. 13015-13024, 2015.

[38] A. Sewaiwar, V. S. Tiwari and H. Y. Chung, "Novel user allocation scheme for full duplex multiuser bidirectional Li-Fi network," Optics Communication, vol. 339, p. 153-156, 2015.
[39] H. Kim, S. Tiwari, and Y. Chung, "Multi-hop relay-based maritime visible light communication," Chinese Optics Letters, vol. 14, pp. 050607 , 2016.

[40] M. C. Kim, I. S. Choi and J. S. Koh, "IDMP-VLC: IoT device management protocol in visible light communication networks," in 19th International Conference on Advanced Communication Technology (ICACT), Bongpyeong, 2017.

[41] X. Guo, M. Mohammad, S. Saha, C. M. Chan, S. Gilbert and D. Leong, "PSync: Visible light-based time synchronization for Internet of Things (IOT)," in IEEE INFOCOM, The 35th Annual IEEE International Conference on Computer Communications, San Francisco, 2016.

[42] S. Ma, Q. Liu and Y. C. P. Sheu, "Foglight: visible light-enabled indoor localization system for low-power IoT devices," IEEE Internet of Things Journal, vol. 5, no. 1, pp. 175-185, 2018.

[43] O. Anilkumar and R. K. Jeyachitra, "Performance enhancement of indoor VLC system by employing SAC OCDMA technique," 2017 International Conference on Wireless Communications, Signal Processing and Networking (WiSPNET), Chennai, 2017, pp. 1784-1789.

[44] W. Huang, P. Tian, and Z. Xu, "Design and implementation of a real-time CIM-MIMO optical camera communication system," Optics Express, vol. 24, pp. 24567-24579, 2016.

[45] A. Sewaiwar, S. V. Tiwari and H. Y. Chung, "Visible light communication based motion detection," Optics Express, vol. 23, no. 14, p. 18769-18776, 2015 .

[46] S. V. Tiwari, A. Sewaiwar, and Y. Chung, "Optical bidirectional beacon based visible light communications," Optics Express, vol. 23, pp. 26551$26564,2015$.

[47] Y. Lecun, Y. Bengio and G. Hintin, "Deep Learning," Nature, vol. 521, pp. 436-444, 2015.

[48] Z. Ghassemlooy, A. P. Haigh, F. Arca, F. S. Tedde, O. Hayden, I. Papakonstantinou and S. Rajbhandari, "Visible light communications: $3.75 \mathrm{Mb} / \mathrm{s}$ data rate with a $160 \mathrm{kHz} \mathrm{BW}$ organic photodetector and ANN equalization," SIOM, Chinese Laser Press, 2012.

[49] A. Aslam and E. Curry, "Towards a generalized approach for deep neural network based event processing for the internet of multimedia things," IEEE Access, in press, 2018.

[50] S. Teli, A. W. Anugrah and H. Y. Y. H. Chung, "Trained neurons-based motion detection in optical camera communications," Optical Engineering, vol. 57, no. 4, 2018.

[51] H. Lee, I. Lee and H. S. Lee, "Deep learning based transceiver design for multi-colored VLC systems," Optics Express, vol. 26, pp. 6222-6238, 2018.

[52] A. P. Haigh, Z. Ghassemlooy, S. Rajbhandari, I. Papakonstantinou and W. Popoola, "Visible light communications: $170 \mathrm{Mb} / \mathrm{s}$ using an artificial neural network equalizer in a low bandwidth white light configuration," Journal of Lightwave Technology, vol. 23, no. 9, pp. 1807-1813, 2014. 